\title{
No-waiting dentine self-etch concept-Merit or hype
}

\author{
Xue-qing Huang ${ }^{\mathrm{a}}$, César R. Pucci ${ }^{\mathrm{b}}$, Tao Luo ${ }^{\mathrm{c}}$, Lorenzo Breschi ${ }^{\mathrm{d}}$, David H. Pashley ${ }^{\mathrm{e}}$, Li-na Niu ${ }^{\mathrm{f}, * *}$, \\ Franklin R. Tay ${ }^{\text {e,* }}$ \\ a Department of Prosthodontics, Guanghua School and Hospital of Stomatology \& Guangdong Provincial Key Laboratory of Stomatology, Sun Yat-sen University, \\ Guangzhou, Guangdong, PR China \\ b Department of Restorative Dentistry, Institute of Science and Technology, São Paulo State University UNESP São Jose dos Campos, São Paulo, Brazil \\ ${ }^{c}$ Key Laboratory of Oral Medicine, Guangzhou Institute of Oral Disease, Stomatology Hospital of Guangzhou Medical University, Guangzhou, Guangdong, PR China \\ d Department of Biomedical and Neuromotor Sciences, DIBINEM, University of Bologna - Alma Mater Studiorum, Bologna, Italy \\ e College of Graduate Studies, Augusta University, Augusta, GA, USA \\ ${ }^{f}$ State Key Laboratory of Military Stomatology \& National Clinical Research Center for Oral Diseases \& Shaanxi Key Laboratory of Oral Diseases, School of Stomatology, \\ The Fourth Military Medical University, Xi'an, Shaanxi, PR China
}

\section{A R T I C L E I N F O}

\section{Keywords:}

Dentine

Self-etch

Time factors

Universal adhesives

\begin{abstract}
A B S T R A C T
Objective: A recently-launched universal adhesive, G-Premio Bond, provides clinicians with the alternative to use the self-etch technique for bonding to dentine without waiting for the adhesive to interact with the bonding substrate (no-waiting self-etch; Japanese brochure), or after leaving the adhesive undisturbed for $10 \mathrm{~s}$ (10-s selfetch; international brochure). The present study was performed to examine in vitro performance of this new universal adhesive bonded to human coronal dentine using the two alternative self-etch modes.

Methods: One hundred and ten specimens were bonded using two self-etch application modes and examined with or without thermomechanical cycling (10,000 thermal cycles and 240,000 mechanical cycles) to simulate one year of intraoral functioning. The bonded specimens were sectioned for microtensile bond testing, ultrastructural and nanoleakage examination using transmission electron microscopy. Changes in the composition of mineralised dentine after adhesive application were examined using Fourier transform infrared spectroscopy.

Results: Both reduced application time and thermomechanical cycling resulted in significantly lower bond strengths, thinner hybrid layers, and significantly more extensive nanoleakage after thermomechanical cycling. Using the conventional 10-s application time improved bonding performance when compared with the nowaiting self-etch technique. Nevertheless, nanoleakage was generally extensive under all testing parameters employed for examining the adhesive.

Conclusion: Although sufficient bond strength to dentine may be achieved using the present universal adhesive in the no-waiting self-etch mode that does not require clinicians to wait prior to polymerisation of the adhesive, this self-etch concept requires further technological refinement before it can be recommended as a clinical technique.

Clinical significance: Although the surge for cutting application time to increase user friendliness remains the most frequently sought conduit for advancement of dentine bonding technology, the use of the present universal adhesive in the no-waiting self-etch mode may not represent the best use of the adhesive.
\end{abstract}

\section{Introduction}

Science and technology constitute a primary productive force that stimulates society reform [1]. As early as the 1700s, technological developments that led to the introduction of steam power catalysed the Industrial Revolution and provided the backdrop for modernisation on a global scale. Technologies triggered by the concepts of packet switching and wide-area networking in the 1960s incited the emergence of the internet and metamorphosed nearly all facets of human activity - society, politics, culture and the economy. On a lighter note, the technology triggered by Doctor Michael Buonocore's adaptation of phosphoric acid treatment of metal surfaces to obtain better adhesion of paint primers spurred the rise of the resin-bonding epoch in dentistry [2].

\footnotetext{
* Corresponding author at: The Dental College of Georgia, Augusta University, Augusta, Georgia, USA.

*** Corresponding author.

E-mail addresses: niulina831013@126.com (L.-n. Niu), ftay@augusta.edu (F.R. Tay).
} 
Table 1

Adhesive composition and application procedures.

\begin{tabular}{|c|c|c|c|}
\hline Adhesive & $\mathrm{pH}$ & Composition & Manufacturer's instructions \\
\hline \multirow[t]{10}{*}{ G-Premio Bond } & 1.5 & $\begin{array}{l}\text { 10-MDP, 4-META, 10-methacryoyloxydecyl dihydrogen thiophosphate, methacrylate acid ester, } \\
\text { distilled water, acetone, phot initiators, silica }\end{array}$ & No-waiting self-etching mode \\
\hline & & & 1. Apply using a microbrush \\
\hline & & & 2. No waiting after applicatio \\
\hline & & & $\begin{array}{l}\text { 3. Dry thoroughly for } 5 \mathrm{~s} \text { with oil-free air under } \\
\text { maximum air pressur }\end{array}$ \\
\hline & & & 4. Light-cure for $10 \mathrm{~s}$ \\
\hline & & & 10-s self-etching mode \\
\hline & & & 1. Apply using a microbrush \\
\hline & & & 2. Leave undisturbed for $10 \mathrm{~s}$ after application \\
\hline & & & $\begin{array}{l}\text { 3. Dry thoroughly for } 5 \mathrm{~s} \text { with oil-free air under } \\
\text { maximum air pressure }\end{array}$ \\
\hline & & & 4. Light-cure for $10 \mathrm{~s}$ \\
\hline
\end{tabular}

Abbreviations. 10-MDP: 10-methacryloxydecyl dihydrogen phosphate; 4-META: 4-methacryloyloxyethyl trimellitic anhydride.

Beginning with the initial technology trigger, the platform associated with the development and coming-of-age of new technologies appears to follow a similar cycle, irrespective of the field of endeavour [3-5]. In the context of dentine bonding, the enthusiasms associated with the advent of simultaneous etching of enamel and dentine [6], multi-step etch-and-rinse adhesives [7] and the water-wet bonding etch-and-rinse technique [8] produced a plethora of successful dental adhesion stories, reaching a peak of inflated expectations during the early 1990s [9]. No sooner had clinicians been besotted with these excitements than reports of post-operative sensitivity and adversarial pulpal responses associated with the use of those adhesives began to surface, particularly when the adhesives were applied to deep, vital dentine $[10,11]$. By etching and priming tooth substrates simultaneously, two-step self-etch adhesives were subsequently introduced to solve those problems by reducing dentine permeability through the incorporation of smear layer components into the hybrid layer [12]. Paradoxically, the problem-solving strategy contributed to unforeseen problems. Increases in water sorption associated with incorporating increasingly hydrophilic adhesive resin monomers [13], adhesive coatings with semi-permeable membrane-like characteristics [14], as well as bond degradation over time $[15,16]$ became the "Achilles heel" of both etch-and-rinse and self-etch adhesives. Waxing and waning of the promises to deliver speedier, easier and more user-friendly adhesive versions precipitated a trough of disillusionment in adhesive dentistry. As more is known about the control of resin-dentine bond degradation via the use of the water-compatible catalytic systems [17], less hydrophilic adhesive resin monomers [16] and inhibition of adhesiveactivated endogenous proteases [16], clinicians begin to appreciate the benefits associated with step reduction for both the etch-and-rinse [7] and self-etch adhesives [18]. The simplified clinical manipulation and shortened application time associated with these new adhesive genres moved the industry to a slope of enlightenment. Universal adhesives emerged as the latest player in this arena [19]. They provided dentists with the option in choosing adhesive strategies (i.e. etch-and-rinse, selfetch or selective etch), the versatility in bonding to zirconia [20] and the purported advantage of bonding to silica-based ceramics without the use of additional primers [21]. Nevertheless, universal adhesives have been perceived as old wines in new bottles [22] because they inherit the limitations of previous adhesive categories [23]. In the absence of further enlightenment, it appears that dentine adhesive development has entered the plateau stage of a classical technology development cycle. New insights are thus required to transcend technological development of dentine adhesives to new heights.

A recently-launched universal adhesive (G-Premio Bond, GC Corporation, Tokyo, Japan) provides the clinician with the alternative to use the self-etch technique for bonding to dentine without waiting for the adhesive to interact with the bonding substrate (no-waiting self- etch; Japanese version of manufacturer's instructions), or after leaving the adhesive undisturbed for $10 \mathrm{~s}$ (10-s self-etch; international version of manufacturer's instructions) [24]. Introduction of the no-waiting concept, while appealing to clinicians, should not compromise adhesive bonding effectiveness and the durability of resin-dentine bonds. Hence, the present study was performed to examine in vitro performance of the single-component, universal adhesive bonded to human coronal dentine using the two aforementioned alternative self-etch modes. Accordingly, two null hypotheses were tested: 1) there is no difference between the use of no-waiting self-etch and 10-s self-etch on the microtensile bond strength of the universal adhesive to dentine; and 2) thermomechanical cycling has no effect on the microtensile bond strength to dentine when the universal adhesive is used in either the nowaiting or the 10 -s self-etch mode.

\section{Materials and methods}

\subsection{Dentine disc preparation}

One hundred and ten freshly extracted intact human third molars were collected according to a protocol approved by the Human Assurance Committee of the Augusta University, with informed consent obtained from the donating subjects with respect to the use of human tissues. The extracted teeth were stored in $0.9 \%(\mathrm{w} / \mathrm{v}) \mathrm{NaCl}$ containing $0.02 \%$ sodium azide at $4{ }^{\circ} \mathrm{C}$ for no longer than one month. The roots of the teeth were removed using a water-cooled low-speed saw (Isomet, Buehler Ltd, Evanston, IL, USA). For each tooth, a flat bonding surface was prepared by removing the occlusal third of each tooth crown to expose the mid-coronal dentine. The dentine surfaces were polished with 320-grit silicon carbide paper under water irrigation for $1 \mathrm{~min}$.

\subsection{Bonding procedures}

The teeth were randomly assigned to two groups (50 teeth per group). The composition of G-Premio Bond (GP) is listed in Table 1. Self-etch bonding procedures were performed according to the Japanese version of the manufacturer's instructions (no-waiting self-etch mode) or the international version of the manufacturer's instructions (10-s self-etch mode). For the no-waiting self-etch mode, the adhesive was applied on the dentine surface and immediately air-dried without waiting (see below). For 10-s self-etch mode, the adhesive was applied on the dentine surface and left undisturbed for $10 \mathrm{~s}$. For both modes, the adhesive was dried for $5 \mathrm{~s}$ using oil-free air from the triple syringe of a clinical operatory unit under maximum air pressure. After light-curing of the adhesive, resin composite build-ups were constructed in four 1$\mathrm{mm}$ thick increments using G-ænial Sculpt (GC Corporation). Each increment was light-cured for $20 \mathrm{~s}$ using a light-emitting diode 
(440-480 nm range) curing unit with an output intensity of $1200 \mathrm{~mW} /$ $\mathrm{cm}^{2}$. To facilitate sectioning of transmission electron microscopy (TEM) specimens, the bonded specimens were coupled with a $2 \mathrm{~mm}$ thick layer of Protect Liner F flowable resin composite (Kuraray Noritake Dental Inc., Tokyo, Japan) and light-cured for $20 \mathrm{~s}$.

Because the manufacturer emphasised the mandatory use of maximum air pressure for drying the adhesive in the bonding instructions, a pilot study was also performed to understand the consequence of not adhering to this instruction. In the pilot study, additional dentine discs were bonded using the two aforementioned self-etch protocols $(\mathrm{N}=2)$, but with the adhesive dried with $5 \mathrm{~s}$ of gentle air flow. After coupling with the flowable resin composite, the bonded specimens were aged for $24 \mathrm{~h}$ and prepared for nanoleakage examination of the resin-dentine interfaces (methods described in the TEM section).

After bonding, the specimens from each experimental group (i.e. those not related to the pilot study) were divided into two batches. One batch was stored in deionised water at $37^{\circ} \mathrm{C}$ for $24 \mathrm{~h}$; the other batch was subjected to thermomechanical challenge, using 10,000 thermal cycles $\left(10{ }^{\circ} \mathrm{C}\right.$ for one min, $25^{\circ} \mathrm{C}$ for one min and $55{ }^{\circ} \mathrm{C}$ for one min) and 240,000 mechanical cycles, corresponding to one year of intraoral functioning $[25,26]$. The resin-dentine specimens were sectioned in both $\mathrm{x}$ and $\mathrm{y}$ directions across the adhesive interface to obtain beams with cross-sectional areas of approximately $0.9 \mathrm{~mm} \times 0.9 \mathrm{~mm}$ using the 'non-trimming' version of the microtensile bond testing procedures [27]. Simulated ageing was performed in a thermomechanical wear system (Model ER-37000; Erios, São Paulo, SP, Brazil). The four longest beams were obtained from the two central slabs of each bonded tooth for bond strength testing and one beam each was obtained from the two central slab of each bonded tooth for TEM. Hence, for each of the four subgroups, 80 beams from 20 teeth were used for bond strength testing and 10 beams from 5 teeth were used for TEM examination of the resindentine interface.

\subsection{Microtensile bond strength}

Each beam from a subgroup $(\mathrm{N}=20)$ was secured with cyanoacrylate glue (Zapit; Dental Ventures of America, Corona, CA, USA) to a testing jig and stressed to failure under tension in a universal testing machine (Vitrodyne V1000; Liveco Inc., Burlington, VT, USA) at a cross-head speed of $1 \mathrm{~mm} / \mathrm{min}$. The tensile load at failure was recorded and divided by the measured cross-sectioned area of each beam to yield the tensile bond strength in megaPascals (MPa). The mean bond strength of the 4 beams derived from one tooth was used to represent the tensile bond strength of that tooth, yielding 20 values per subgroup. Data were analysed with parametric statistical methods after validating the normality (Shapiro-Wilk test) and homogeneity of variance (modified Levene test) assumptions of the data sets. A two-factor analysis of variance was used to examine the effects of adhesive application time (i.e. no-waiting or 10-s self-etch) and simulated ageing (i.e. without or with thermomechanical cycling), and the interaction of those two factors on the bond strength results. Post-hoc pairwise comparisons were performed using the Tukey statistic. Statistical significance was set at $\alpha=0.05$.

After bond strength testing, the two ends of a fractured stick were retrieved and examined with $10 \times$ magnification using a stereoscopical microscope to determine the mode of failure. Failure modes were classified as adhesive failure (failure along the adhesive interface), mixed failure (failure within the adhesive joint with failure within the resin composite or dentine), or cohesive failure (failure within the resin composite or dentine). For statistical analysis, the number of specimens exhibiting mixed and cohesive failures were combined into the nonadhesive category. Adhesive and non-adhesive failure modes in the 4 subgroups (zero-second or 10-s etch; with or without thermomechanical cycling) were arranged into a $2 \times 4$ contingency table and analysed with the Fisher-Freeman-Halton statistic [28]. A 95\% confidence level was used to determine if an association existed between the method in which the specimens were bonded and tested, and the category of failure (adhesive $v s$ non-adhesive).

\subsection{Transmission electron microscopy}

Five of the 10 beams designated for TEM were used for nanoleakage examination. Each beam was coated with two layers of nail varnish applied to within $1 \mathrm{~mm}$ of the bonded interface. After drying, the varnish-coated beams were immersed in 50\% ammoniacal silver nitrate solution for 48 hours. The silver-impregnated specimens were thoroughly rinsed with deionised water and placed in photo-developing solution for $8 \mathrm{~h}$, under a fluorescent light, to facilitate reduction of the diaminesilver ions into metallic silver grains [29]. The silver-impregnated specimens were polished, cleaned ultrasonically, dehydrated in an ascending ethanol series (50-100\%), immersed in propylene oxide as transition medium, and embedded in epoxy resin. Ninety nanometrethick sections were prepared using an ultramicrotome and examined without staining, using a JEM-1230 TEM (JEOL, Tokyo, Japan) at $110 \mathrm{kV}$.

For each beam, 5 images taken at $10,000 \times$ magnification were analysed with the Scion Image software (Scion Corp., Frederick, MD) for the percentage area within the resin-dentine interface (i.e. the adhesive layer and hybrid layer) occupied by the silver tracer. The mean of the percentage of interface containing silver deposits from the 5 images was used to represent the overall nanoleakage of a subgroup. Because the homogeneity of variance assumption of the data sets derived from the 4 subgroups was violated, the interval data (in percentages) were logarithmically transformed to satisfy both normality and equal variance assumptions. The transformed data were analysed with two-factor analysis of variance to examine the effects of adhesive application time and simulated ageing, and the interaction of those two factors on the nanoleakage results. Post-hoc pairwise comparisons were performed using the Tukey statistic. Statistical significance was set at $\alpha=0.05$.

The other five beams from each subgroup were completely demineralised in $0.1 \mathrm{M}$ formic acid/sodium formate $(\mathrm{pH} 2.5)$. The end point of demineralisation was determined by drop-wise addition of a $10 \%$ potassium oxalate solution to the demineralisation medium, which formed a white calcium oxalate precipitate when calcium ions were present. Completely demineralised beams containing the resin-dentine interface were fixed with Karnovsky's fixative (2.5 wt $\%$ glutaraldehyde and $2 \%$ paraformaldehyde in $0.1 \mathrm{~mol} / \mathrm{L}$ cacodylate buffer; $\mathrm{pH}, 7.3$ ) for $8 \mathrm{~h}$, and post-fixed in $1 \%$ osmium tetroxide for $1 \mathrm{~h}$. The fixed specimens were dehydrated in an ascending ethanol series (30-100\%), immersed in propylene oxide as a transition medium and ultimately embedded in pure epoxy resin. Ninety-nanometre thick sections were prepared, stained with $2 \%$ aqueous uranyl acetate and Reynold's lead citrate, and examined using the JEM-1230 TEM at $110 \mathrm{kV}$.

\subsection{Attenuated total reflection-Fourier transform infrared spectroscopy}

A Nicolet 6700 spectrophotometer (Thermo Scientific Inc., Waltham, MA, USA) with an attenuated total reflection setup was used to collect infrared spectra from dentine discs before self-etching (control), and after etching the mineralised dentine without waiting or for $10 \mathrm{~s}(\mathrm{~N}=2$ dentine discs). The universal adhesive was applied respectively to each disc without light-curing. The etched dentine surface was rinsed with absolute ethanol to completely remove the uncured adhesive prior to scanning. The rationale of adhesive dissolution was to prevent interference of the adhesive vibrational peaks with the peaks characteristic of mineralised dentine. Spectra were collected between 4000 and $500 \mathrm{~cm}^{-1}$ at $4 \mathrm{~cm}^{-1}$ resolution using 32 scans. The spectra were superimposed after correction of their baseline shifts, so that the intensities of the carbonated apatite-associated peaks could be compared. 


\section{Without thermomechanical cycling After thermomechanical cycling}

\section{A Microtensile bond strength}
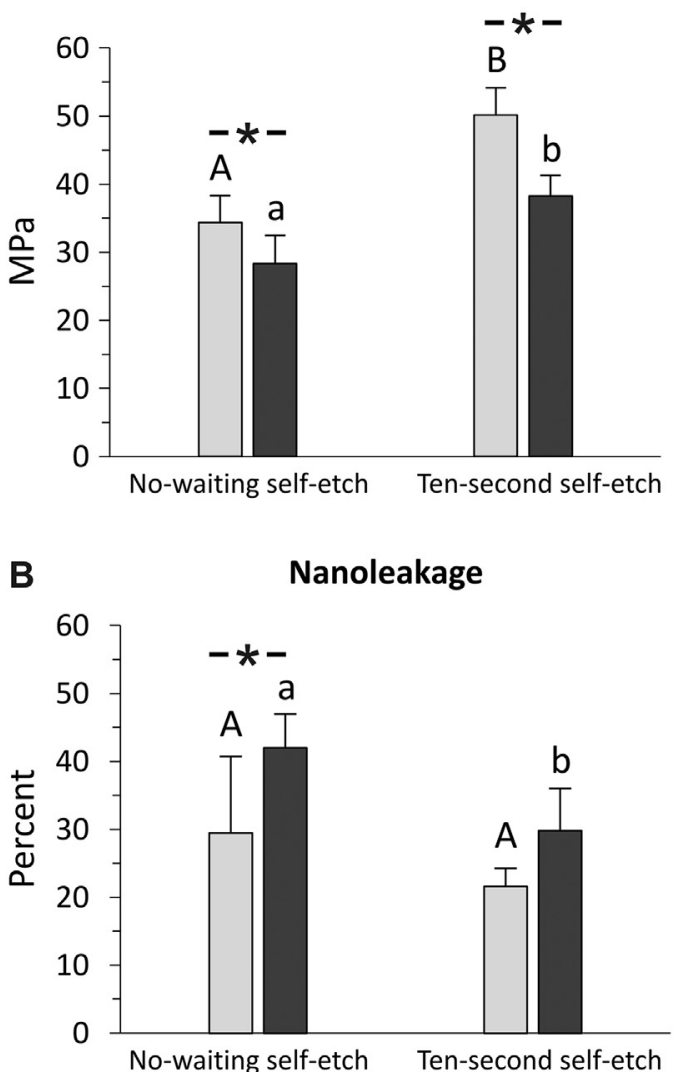

Fig. 1. Comparison of A. microtensile bond strength (in MPa), and B. nanoleakage (in percentages) of the universal adhesive (G-Premio Bond, GC Corp., Tokyo, Japan) bonded to dentine using the no-waiting or the 10-s self-etching mode, with or without thermomechanical cycling. For each chart, data represent mean values and standard deviations. for the factor "adhesive application time", subgroups labelled with different letter superscripts (upper case for without cycling and lower case for after cycling) are significantly different $(\mathrm{P}<0.01)$. For the factor "simulated ageing", subgroups connected by a bar with asterisk are significantly different $(\mathrm{P}<0.01)$

\section{Results}

Microtensile bond strengths of the four subgroups bonded with the universal adhesive (no-waiting self-etch, 10-s self-etch, with or without thermomechanical cycling) are summarised in Fig. 1A. Bond strength was significantly affected by the adhesive application time $(\mathrm{P}<0.001)$ and whether simulated ageing was performed $(\mathrm{P}<0.001)$. There was a statistically significant interaction between the two factors $(\mathrm{P}=0.001)$. For pairwise comparisons within the factor adhesive application time, bond strength of the no-waiting self-etch subgroup $(34.4 \pm 3.9 \mathrm{MPa})$ was significantly lower $(\mathrm{P}<0.001)$ than the 10-s self-etch subgroup (50.2 $\pm 4.0 \mathrm{MPa})$ when thermomechanical cycling was not performed. Likewise, bond strength of the no-waiting self-etch subgroup $(28.3 \pm 4.1 \mathrm{MPa})$ was significantly lower $(\mathrm{P}<0.001)$ than the 10-s self-etch subgroup $(38.3 \pm 3.0 \mathrm{MPa})$, after the specimens were thermomechanically cycled. In both the no-waiting self-etch subgroup and the 10-s self-etch subgroup, the bond strength decreased significantly after thermomechanical cycling $(\mathrm{P}<0.001)$. For pairwise comparisons within the factor simulated ageing, significant differences were identified between the no-waiting and 10-s selfetch subgroups irrespective of whether thermomechanical cycling was performed ( $\mathrm{P}<0.001$ for both pairwise comparisons).
Table 2

$2 \times 4$ contingency table showing the distribution of adhesive and non-adhesive (i.e. mixed and cohesive combined) failures in the 4 subgroups.

\begin{tabular}{llllll}
\hline & $\begin{array}{l}\text { No-waiting } \\
\text { self-etch } \\
\text { No TMC }\end{array}$ & $\begin{array}{l}\text { No-waiting } \\
\text { self-etch } \\
\text { After TMC }\end{array}$ & $\begin{array}{l}\text { 10-s } \\
\text { self-etch } \\
\text { No TMC }\end{array}$ & $\begin{array}{l}\text { 10-s } \\
\text { self-etch } \\
\text { TMC }\end{array}$ & Totals \\
\hline $\begin{array}{c}\text { Adhesive } \\
\text { failure }\end{array}$ & 15 & 19 & 12 & 18 & 64 \\
$\begin{array}{c}\text { Non-adhesive } \\
\text { failure }\end{array}$ & 5 & 1 & 8 & 2 & 16 \\
\begin{tabular}{c} 
Totals \\
\hline
\end{tabular} & 20 & 20 & 20 & 20 & 80 \\
\hline
\end{tabular}

Abbreviation: TMC: thermomechanical cycling

Fisher-Freeman-Halton statistic

$P_{\mathrm{A}}=\mathbf{0 . 0 3 5 4}$, where

$\mathrm{P}_{\mathrm{A}}$ : the probability of the observed array of cell frequencies plus the sum of the probabilities of all other cell-frequency arrays (such as would be consistent with the observed marginal totals) that are $\leq$ the probability of the observed array.

$\mathbf{P}_{\mathrm{B}}=\mathbf{0 . 0 2 9 1}$, where

$\mathrm{P}_{\mathrm{B}}$ : the probability of the observed array of cell frequencies plus the sum of the probabilities of all other cell-frequency arrays (such as would be consistent with the observed marginal totals) that are $<$ the probability of the observed array.

Number of tables evaluated $=969$

The chi-square test was not performed because more than $20 \%$ of the cells have an expected frequency of less than 5 .

Failure mode analysis for all subgroups indicates that more than $60 \%$ of the specimens exhibited adhesive failure, while the remaining were mixed failure or cohesive failure in resin or dentine. Because only a few incidents of cohesive failure were identified in all subgroups, cohesive failures were combined with mixed failures in each subgroup (i.e. non-adhesive failures) for statistical analysis. A $2 \times 4$ contingency table depicting the frequency of adhesive and non-adhesive failures in the beams derived from the 4 subgroups is shown in Table 2. The sum of the probabilities of the observed array of cell frequencies, together with the probabilities of all other cell-frequency arrays that are less than or equal to the probability of the observed array (i.e. $\mathrm{P}_{\mathrm{A}}$ ) is 0.0354 . There was a statistically significant association between the method in which the specimens were bonded and tested, and the category of failure $(\mathrm{P}<0.05)$.

The ultrastructure of the resin-dentine interface and representative images of nanoleakage that occurred within the interface of specimens bonded using the no-waiting self-etch mode are shown in Fig. 2 (no thermomechanical cycling) and Fig. 3 (after thermomechanical cycling). The hybrid layers were generally less than $200 \mathrm{~nm}$ thick in most of the sections, spanning the width of two layers of collagen fibrils (Fig. 2, left column). The thickness of the hybrid layer was uneven in some sections and there were regions where the hybrid layer was very thin or missing (Fig. 3, left column), with occasional remnants of the smear layer. Despite the application of a single coat of adhesive (3-5 $\mu \mathrm{m}$ thick), the basal portion of the polymerised, filled adhesive that approximated the hybrid layer and dentinal tubules was more electron-dense after staining with the water-based heavy metal stains, when compared with the coronal portion of the stained adhesive. In unstained, non-demineralised sections (right column of both figures), nanoleakage was manifested as extensive patches of electron-dense, reticular silver deposits that were the most extensive at the base of the adhesive layer above the hybrid layer. Specimens after thermomechanical cycling exhibited almost continuous silver tracer infusion (Fig. 3) along the base of the adhesive layer, when compared with specimens that had not been subjected to thermomechanical cycling (Fig. 2). Although extensive nanoleakage was observed, all the intensely airdried specimens (i.e. maximum air pressure for $5 \mathrm{~s}$ ) were devoid of water bubble entrapment within the adhesive layer. In contrast, abundant silver-impregnated water bubbles could be identified from the thicker adhesive layers ( $>10 \mu \mathrm{m}$ thick) present in the pilot study specimens which were dried with gentle air pressure only (Supplementary information Fig. S-1). 
Demineralised, stained

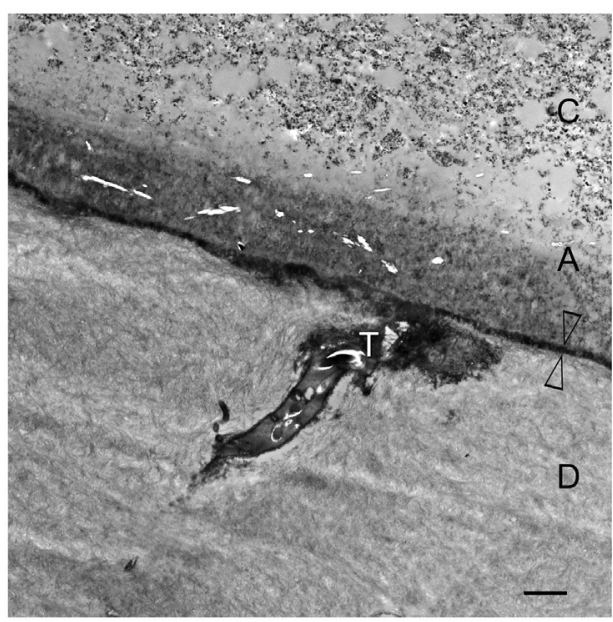

Mineralised, unstained, silver-infiltrated

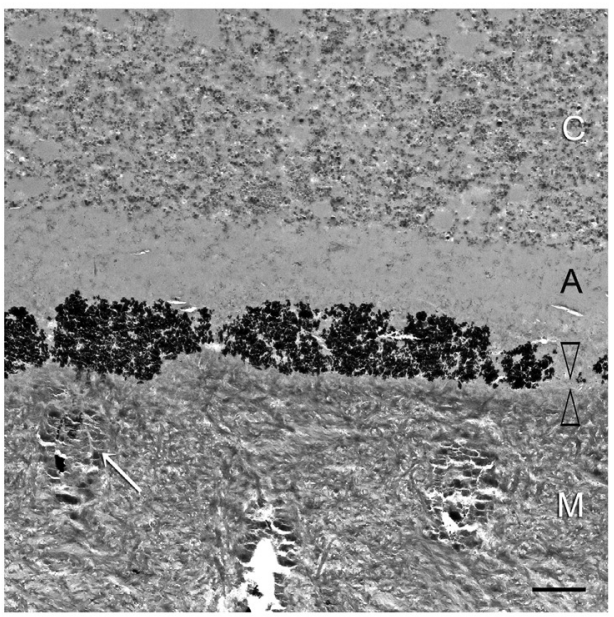

A.

Bar $=2 \mu \mathrm{m}$
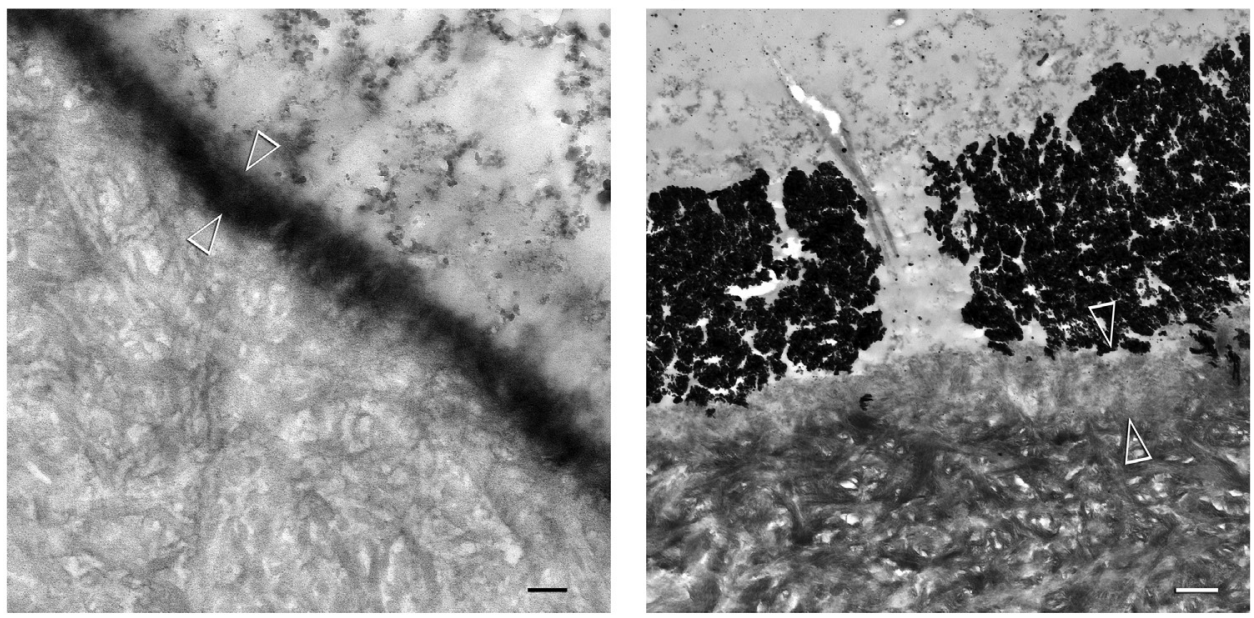

\section{Bar $=200 \mathrm{~nm}$}

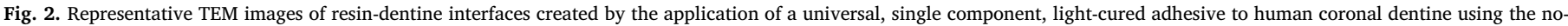

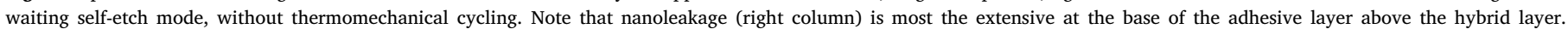
Abbreviations: Afilled adhesive ; Between open arrowheadshybrid layer ; Cresin composite ; Ddemineralised dentine ; Mmineralised dentine ; Tdentinal tubule.

The ultrastructure of the resin-dentine interface and representative images of nanoleakage that occurred within the interface in specimens bonded using the 10-s self-etch mode are shown in Fig. 4 (no thermomechanical cycling) and Fig. 5 (after thermomechanical cycling). In stained, demineralised sections (left columns), electron-dense hybrid layers that were generally $500 \mathrm{~nm}$ thick could be identified. Similar to the no-waiting self-etch subgroups, the adhesive layer was approximately 3-6 $\mu \mathrm{m}$ thick. Although only a single coat of adhesive was applied, a lightly-stained upper layer and a more heavily-stained lower layer were apparent after the polymerised adhesive was stained with uranyl acetate and lead citrate. In unstained, non-demineralised sections (right columns), nanoleakage in both subgroups was manifested as discontinuous islands of reticular silver deposits within the hybrid layer and the basal part of the adhesive layer.

The percentage distributions of nanoleakage with the adhesive and hybrid layers of the four subgroups are depicted in Fig. 1B. The extent of nanoleakage was significantly affected by the adhesive application time $(\mathrm{P}=0.006)$ as well as whether simulated ageing was performed $(\mathrm{P}=0.004)$. There was no statistically significant interaction between the two factors $(\mathrm{P}=0.501)$. For the factor adhesive application time, significant difference was identified between the no-waiting and the 10$\mathrm{s}$ self-etch modes $(\mathrm{P}=0.006)$. Pairwise comparisons indicated that there was no significant difference in the nanoleakage expression from specimens bonded with the two self-etch modes when thermomechanical cycling was not performed $(\mathrm{P}=0.906)$; however, there was significantly more nanoleakage in specimens bonded with the nowaiting self-etch mode after the specimen were thermomechanically cycled $(P=0.015)$. For the factor simulated ageing, thermomechanical cycling had a significant influence on the extent of nanoleakage expression $(\mathrm{P}=0.005)$. Pairwise comparisons indicated that nanoleakage of specimens bonded with the no-waiting self-etch mode were significantly higher after thermomechanical cycling $(P=0.012)$; by contrast, nanoleakage in specimens bonded with the 10-s self-etch mode was not significantly increased after thermomechanical cycling $(\mathrm{P}=0.083)$.

Fig. 6 shows superimposed infrared spectra generated from dentine discs without adhesive application (control), and after the adhesive was applied in the no-waiting or 10-s self-etch mode, followed by dissolution of the adhesive with ethanol. When no adhesive was applied, the mineralised dentine exhibited intense apatite-associated phosphate bands at $\sim 1000-1100 \mathrm{~cm}^{-1}$ (v3 asymmetric P-O stretch), $\sim 560-600 \mathrm{~cm}^{-1}$ (v4 asymmetric O-P-O bending) and $\sim 960 \mathrm{~cm}^{-1}$ (v1 symmetric P-O stretch) [30,31]. Carbonate bands characteristic of carbonated apatite could be detected at $\sim 875 \mathrm{~cm}^{-1}$ (v2 out-of-plane 

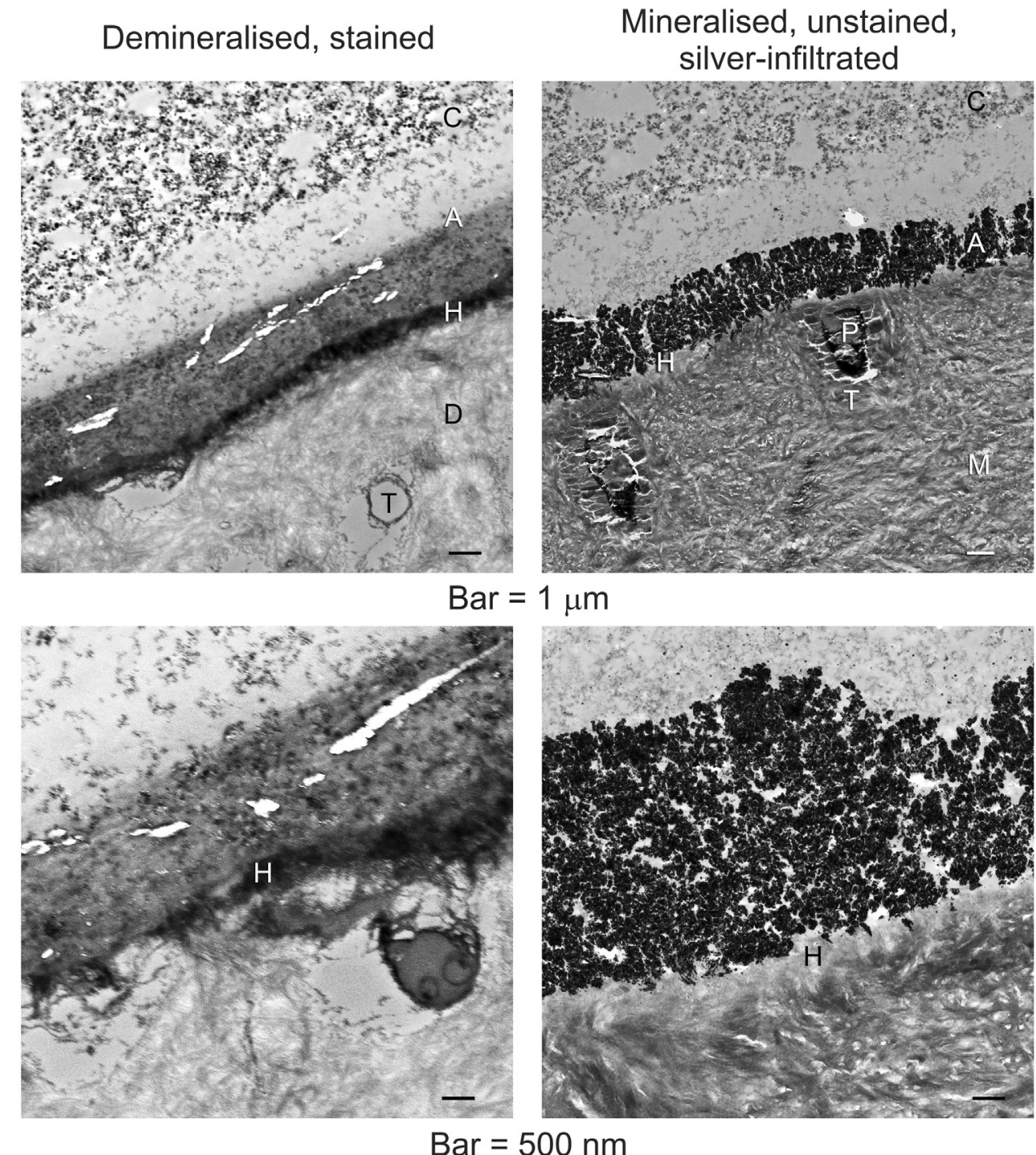

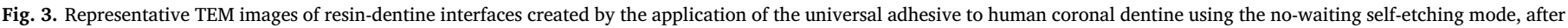

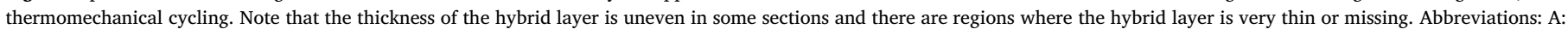
filled adhesive; C: resin composite; D: demineralised dentine; H: hybrid layer; M: mineralised dentine; P: smear plug; T: dentinal tubule.

$\mathrm{O}-\mathrm{C}-\mathrm{O}$ bend) and between $\sim 1440-1550 \mathrm{~cm}^{-1}$ (v3 asymmetric $\mathrm{C}-\mathrm{O}$ stretch) [31]. The intensities of these bands decreased gradually with increases in adhesive application time.

\section{Discussion}

Significantly lower microtensile bond strengths were identified when the adhesive was used in the no-waiting self-etch mode, when compared with using the universal adhesive in the 10-s self-etch mode. This warrants rejection of the first null hypothesis that "there is no difference between the use of no-waiting self-etch and 10-s self-etch on the bond strength to dentine". The use of simulated ageing resulted in reduced bond strengths for the universal adhesive irrespective of application time. Thus, the second null hypothesis that "thermomechanical cycling has no effect on the microtensile bond strength to dentine when the universal adhesive is used in either the no-waiting or the 10-s self-etch mode" also has to be rejected.

Adhesives used in the self-etch mode are designed to bond to tooth substrates by simultaneous etching and resin replacement of the dissolved mineral component [12]. The ability of these adhesives to etch through the smear layer and hybridise the underlying dentine is a time-dependent process that depends on both the aggressiveness of the self-etch adhesive and the thickness of the smear layer [32-34]. In the present work, 320-grit abrasive papers were used to create smear layers with thickness approximating those created by tungsten carbide burs [33]. Transmission electron microscopy of specimens in the no-waiting self-etch, no thermomechanical cycling group (Fig. 2) showed that there was inadequate etching of the smear layer-covered dentine by the acidic monomers within the limited time frame. This probably accounted for the lower microtensile bond strengths obtained with the no-waiting self-etch mode (Fig. 1A). The present result differs from that supplied by the manufacturer http://www.gcamerica.com/ products/operatory/G-Premio_BOND/GCA_G-Premio_BOND_SellSheet11-04-2016-iPad.pdf). The manufacturer claimed that there is no difference in bonding performance between no-waiting (0-s) and 10-s self-etch mode. However, the manufacturer did not reveal details on the method of dentine preparation. For example, the thickness of the smear layer might have been different from that created in the present study. Such an issue is important especially when adhesives are applied with reduced time [24]. G-Premio Bond is a moderately aggressive self-etch adhesive because of its low pH value (1.5; Table 1) [24]. Even though the manufacturer advocates "no-waiting" in its Japanese version of instructions, 2-3 s will have elapsed during the period of adhesive application before the operator begins to evaporate the solvent. This 


\section{Demineralised, stained}

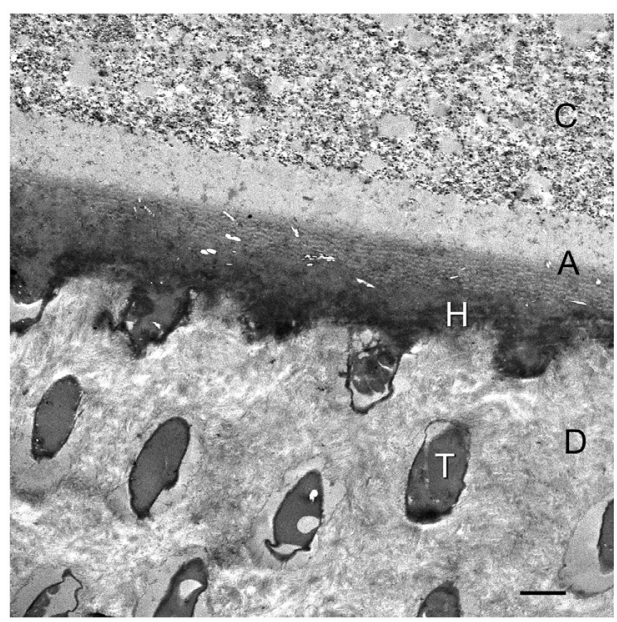

Mineralised, unstained, silver-infiltrated

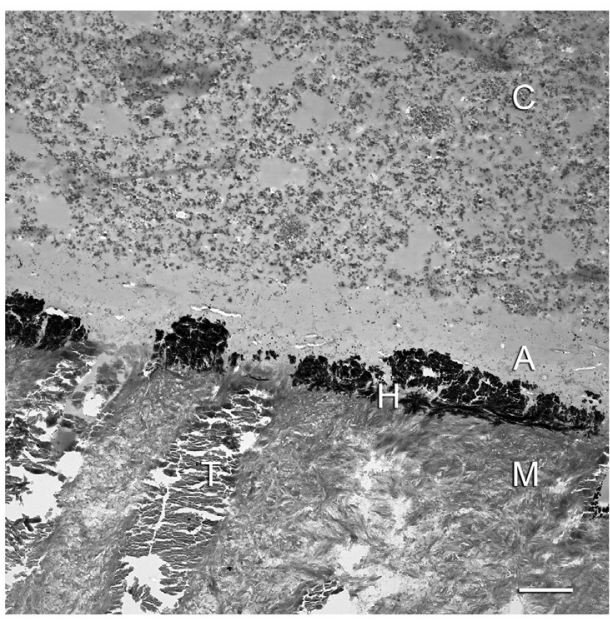

Bar $=2 \mu \mathrm{m}$
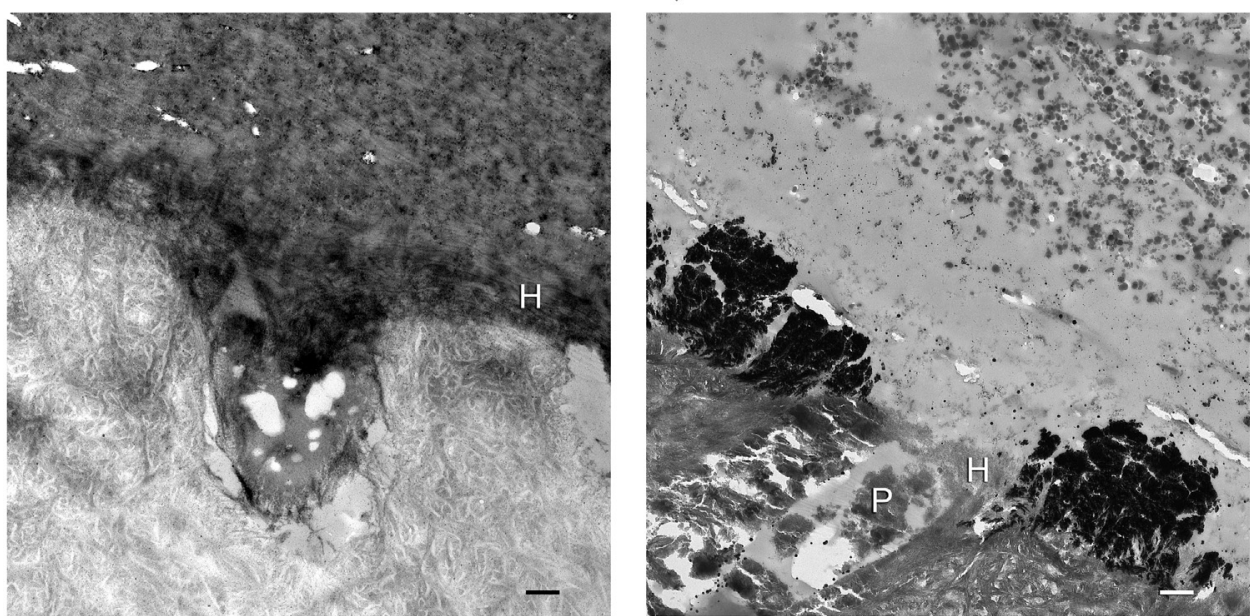

Bar $=500 \mathrm{~nm}$

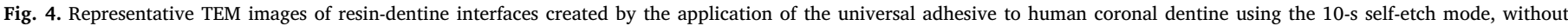

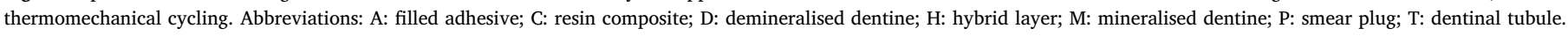

provides a narrow window of opportunity for the adhesive to etch through the smear layer and demineralise the underlying dentine to produce a shallow and uneven hybrid layer (Fig. 3). When application time was increased to $10 \mathrm{~s}$, thicker and more uniform hybrid layers were observed (Fig. 4). Information derived from TEM examination was also supported the infrared spectroscopy data (Fig. 6). The intensities of apatite-associated phosphate bands and carbonate bands decreased with increases in adhesive application time.

Self-etch and universal adhesives contain hydrophilic and hydrophobic resin monomer blends dissolved in miscible solvents [22]. When these water-containing adhesives are formulated without using 2hydroxyethyl methacrylate (HEMA) as a co-solvent, microscopical and even macroscopical phase separation of water-immiscible resin monomers readily occurs after solvent evaporation [35]. For example, phase separation in G-Bond (GC Corporation) is manifested as large globules of water-immiscible resins that are randomly dispersed within the adhesive layer; the phenomenon arises when the adhesive is used against the manufacturer's recommendation in using strong air-blow during the bonding procedures [36,37]. As the latest successor of GBond, G-Premio Bond inherits the same feature and requires strong airblow to reduce microscopical phase separation. Silver-filled water bubbles could be identified within the adhesive layer when only gentle air-blow was used in the pilot study (Supplementary information Fig. S-
1). Evaporation of acetone causes water derived from the adhesive or the underlying dentine to be trapped as water bubbles within the waterimmiscible adhesive components [38]. Evaporation of the adhesive solvent with strong air-blasts removed the bulk of the interfacial water, leaving adhesive layers free of water droplets (Figs. 2-5). Even when microscopical phase separation is eliminated with strong air-blow, separation of the adhesive may occur at the nanoscale after solvent evaporation. Nanoscopical phase separation has been described as undesirable flaws in adhesive design that creates hydrophilic resinous domains that are susceptible to expedited degradation by salivary esterases $[39,40]$. In the present context, although only a single coat of adhesive was applied, a less electron-dense coronal adhesive zone and a more electron-dense basal adhesive zone were apparent after the resin-dentine interface was stained with uranyl acetate and lead citrate (Figs. 2-5). Application of polymer hydrogels for extraction of uranium from sea water as an environmental conservation measure sheds light on the possible mechanism that produces this phenomenon. Uptake of uranyl cations is augmented as the hydrophilicity of the polymer increases, due to faster diffusion and adsorption of aqueous solutions into a hydrophilic polymer, compared with a hydrophobic polymer $[41,42]$. In the present context, a diffusion gradient for the uranyl acetate stain was probably created within the adhesive layer based on the differential quantity of hydrophilic resin components across the 


\section{Demineralised, stained}

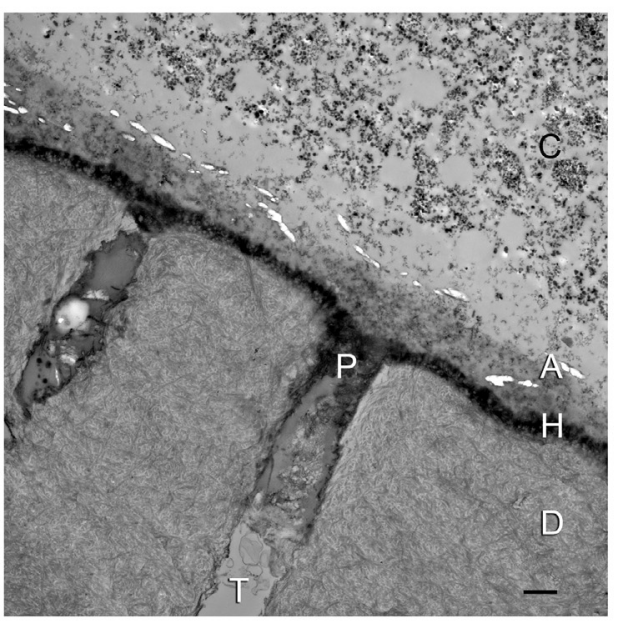

Bar $=1 \mu \mathrm{m}$

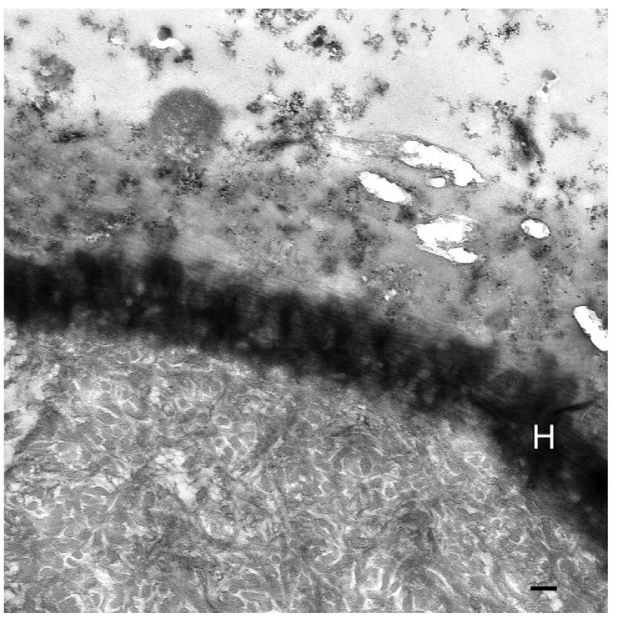

Bar $=200 \mathrm{~nm}$

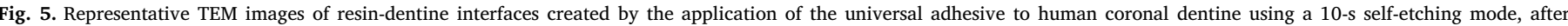

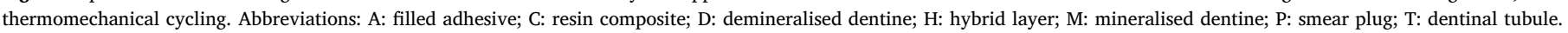

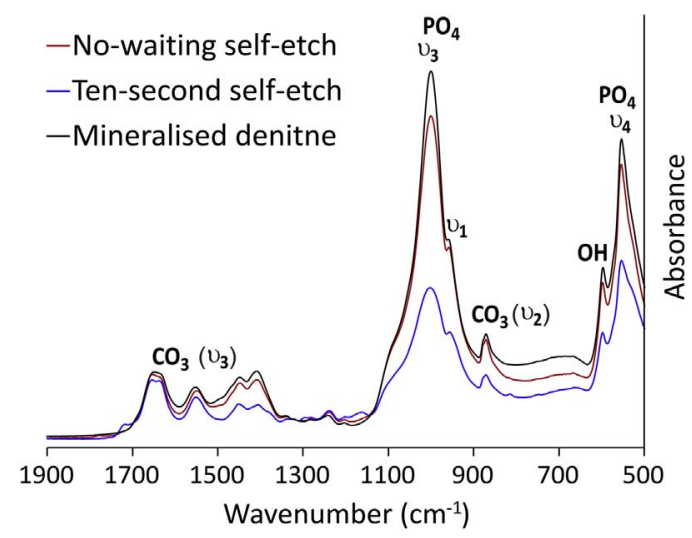

Fig. 6. Representative infrared spectra collected from the surface of mineralised dentine discs without adhesive application (control), and from dentine discs that had been etched with the universal adhesive without waiting or for $10 \mathrm{~s}$. The adhesive was removed prior to spectrum acquisition. The spectra were superimposed after correction of the baseline shifts, so that the intensities of the carbonated apatite-associated peaks may be compared.

thickness of the adhesive layer. The more heavily-stained adhesive zone on top of hybrid layer probably contained more hydrophilic components, whilst the less heavily-stained coronal zone contained more

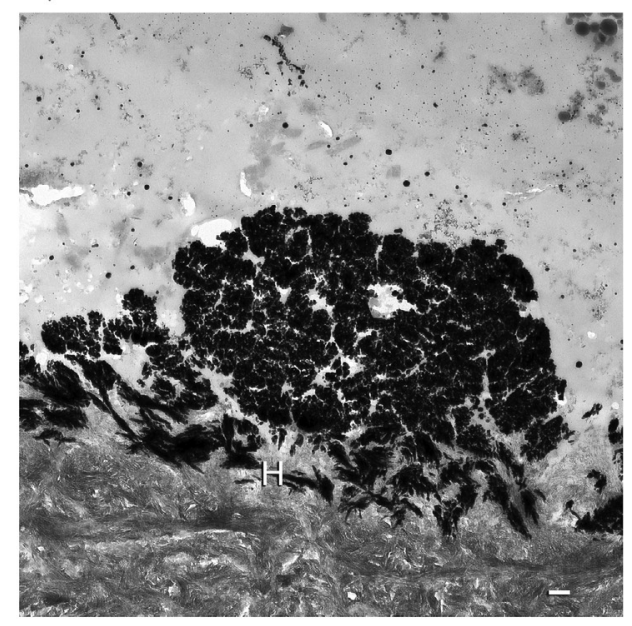

\section{Mineralised, unstained, silver-infiltrated}

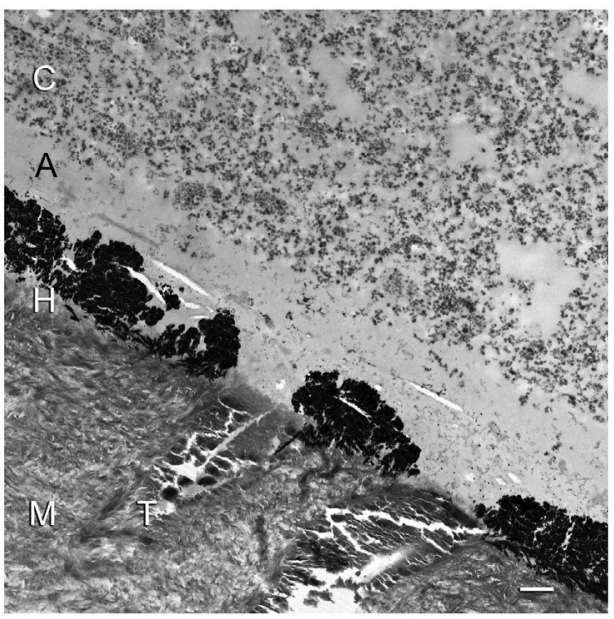


hypertonic adhesive is dispensed, it would extract water/dentinal fluid from the host dentine into the adhesive, producing water trees within the polymerised adhesive [46]. Even in the presence of smear plugs (Fig. 4), slow convective water flux permits water/dentinal fluid to permeate the relatively viscous adhesive following evaporation of the adhesive solvent [48]. In addition, a thick/porous smear layer acts as a water reservoir on the dentine surface that enables water accumulation within the adhesive layer [49]. Collectively, these different types of water fluxes are responsible for the extensive nanoleakage associated with the use of either self-etch mode.

Bond durability of adhesives is critical for clinical success of direct and indirect bonded restorations. Thermal, mechanical or thermomechanical cycling may be conducted to simulate changes in materials caused by intraoral functioning and ageing [50]. A recent study examined the long-term tensile bond strength of resin composite fillings performed in real life and under simulated ageing conditions to identify parameters that may be used to forecast the durability of resin-dentine bonds [51]. Based on the results of that study, a thermomechanical cycling protocol consisting of 10,000 thermal cycles and 240,000 mechanical cycles was used in the present study, corresponding to one year of intraoral functioning [30,31]. This dynamic testing procedure also enables extraction of poorly-polymerised resin oligomers from the resin-dentine interface [39]. This accounts for the increase in the propensity of silver impregnation along the interface after thermomechanical cycling (Fig. 1B) [26]; the phenomenon was more pronounced when the adhesive was applied with reduced application time.

Admittedly, it is not usual to test only one adhesive without comparing the bonding performance with other commercial adhesives to minimise product bias. This may be regarded as the limitation of the present study. An attempt was made to compare G-Premio Bond with two other universal adhesives (Supplementary information, Table S-1). The results indicate that prior to thermomechanical cycling, the bond strength for the G-Premio Bond used in the 10-s self-etch is not significantly different from the other two commercially available universal adhesives. Because G-Premio Bond is currently the only product available in the market in which an adhesive is recommended by the manufacturer to be used in the no-waiting self-etch mode, the most appropriate solution to test the hypotheses in the present study was to use G-Premio Bond in the 10-s self-etch mode as its own control. Revisit of this issue is necessary when similar products are available in the future. Although the thermomechanical cycling regime employed in present study has been reported to approximate 1 year of physiological ageing in the oral cavity, there is no literature to support that thermomechanical cycling alone is sufficient to activate matrix-bound matrix metalloproteinases and cysteine cathepsins present in human dentine [52]. Hence, in-situ zymography as well as long-term waterageing studies are required to evaluate the resistance of resin-dentine interfaces created by the two self-etch modes to degradation by adhesive-activated endogenous proteases [53].

Beginning with the acid-etching trigger that was initiated more than six decades ago, technological developments in dentine bonding have witnessed a "peak of inflated expectations" and subsequently a "trough of disillusionment", before reaching the "slope of enlightenment" and eventually the "plateau of productivity". These phenomena are just about true of the progress of any major technology, as summarised by the Amara's law, which states that "we tend to overestimate the effect of a technology in the short run and underestimate the effect in the long run" [54]. Dentine bonding has progressed to the stage where it normalises with best clinical practices and becomes an expectation. It is thoughtprovoking that the surge for cutting application time to increase user friendliness remains the most frequently sought conduit for advancement of this technology. The pursuit of low hanging fruit represents a manufacturer's business obligation to fulfil consumers' demands, and is brought to dénouement with the advent of the so-called no-waiting dentine self-etch concept. Prior to diving head first into this technolo- gical wave, it is prudent to recollect that the benefits associated with low hanging fruit style innovations are likely to be over-estimated. For innovation to take root in any technological advancement, it must focus on the long-term opportunities where unique ideas and value propositions prevail. Whereas low hanging fruits are tempting and easy to pick, true rewards ultimately come from the willingness to climb higher and stretch for the real treasure. In the context of dentine bonding, realistic challenges remain atop in the arena of improving bond durability. Only when these challenges are met can users of dentine adhesives expect to find real value from innovation.

\section{Conclusion}

Within the limits of the present study, it may be concluded that sufficient short-term bond strength to dentine may be achieved using the universal adhesive investigated in the no-waiting self-etch mode technique prior to polymerisation of the adhesive. Nevertheless, extended application time with the 10-s self-etch mode improves its short-term bonding performance. In addition, thermomechanical cycling decreases the bond strength of the adhesive system when dentine is bonded with either of the two self-etch modes.

\section{Acknowledgements}

This work was supported by National High Technology Research and Development Program of China grant 2015AA020942, National Nature Science Foundation of China grant 81400555 and Natural Science Basic Research Plan in Shaanxi Province of China grant 2015JM8383 (PI: Li-na Niu, The Fourth Military Medical University), National Nature Science Foundation of China grant 81500883 and grant 2015A030401035 from Guangdong Public Research and Competence Special Fund Expenditure (PI: Xue-qing Huang, Sun Yatsen University).

\section{Appendix A. Supplementary data}

Supplementary data associated with this article can be found, in the online version, at 10.1016/j.jdent.2017.05.007.

\section{References}

[1] P. Deng, Science and technology constitute a primary productive force, The Selected Works of Deng Xiao Peng, (2008) (Accessed 04 December 2017), https:// dengxiaopingworks.wordpress.com/2013/03/18/science-and-technologyconstitute-a-primary-productive-force/.

[2] N.V. Babu, R. Joseph, Dr. michael buonocore - 'Adhesive dentistry - 1955', J. Conserv. Dent. 8 (2005) 43-44.

[3] C. Mason, E. Manzotti, Induced pluripotent stem cells: an emerging technology platform and the Gartner hype cycle, Regen. Med. 4 (2009) 329-331.

[4] A.T. Johnson, The technology hype cycle, IEEE. Pulse. 6 (2015) 50.

[5] A.A. Ronfard, M.H. Vertès, A. May, M.E. Dupraz, Y. Bayon van Dyke, Evaluating the past, present, and future of regenerative medicine: a global view, Tissue Eng. Part B Rev. 23 (2017) 199-210.

[6] T. Fusayama, M. Nakamura, N. Kurosaki, M. Iwaku, Non-pressure adhesion of a new adhesive restorative resin, J. Dent. Res. 58 (1979) 1364-1370.

[7] D.H. Pashley, F.R. Tay, L. Breschi, L. Tjäderhane, R.M. Carvalho, M. Carrilho, A. Tezvergil-Mutluay, State of the art etch-and-rinse adhesives, Dent. Mater. 27 (2011) 1-16.

[8] J. Kanca, Improved bond strength through acid-etching of dentin and bonding to wet dentin surfaces, J. Am. Dent. Assoc. 123 (1992) 35-43.

[9] R. Bertolotti, Total etch total seal, total success, Dent. Dimens. 23 (1990) 3-4.

[10] D.H. Pashley, The effects of acid-etching on the pulpodentin complex. Buonocore Memorial Lecture, 1992, Oper. Dent. 17 (1992) 229-242.

[11] E. Asmussen, E.C. Munksgaard, Adhesion of restorative resins to dentinal tissues, in: G. Vanherle, D.C. Smith (Eds.), Posterior Composite Resin Dental Restorative Materials, Peter Szule Publishing Co., 1985, p. 228.

[12] B. Van Meerbeek, J. De Munck, Y. Yoshida, S. Inoue, M. Vargas, P. Vijay, K. Van Landuyt, P. Lambrechts, G. Vanherle, Buonocore memorial lecture. Adhesion to enamel and dentin: current status and future challenges, Oper. Dent. 28 (2003) 215-235.

[13] F.R. Tay, D.H. Pashley, Have dentin adhesives become too hydrophilic? J. Can. Dent. Assoc. 69 (2003) 726-731.

[14] F.R. Tay, D.H. Pashley, B.I. Suh, R.M. Carvalho, A. Itthagarun, Single-step adhesives 
are permeable membranes, J. Dent. 30 (2002) 371-382.

[15] Y.L. Liu, L. Tjäderhane, L. Breschi, A. Mazzoni, N. Li, J. Mao, D.H. Pashley, F.R. Tay, Limitations in bonding to dentin and experimental strategies to prevent bond degradation, J. Dent. Res. 90 (2011) 953-968.

[16] L. Tjäderhane, Dentin bonding: can we make it last? Oper. Dent. 40 (2015) 4-18.

[17] F. Abedin, B. Roughton, Q. Ye, P. Spencer, K. Camarda, Computer-aided molecular design of water compatible visible light photosensitizers for dental adhesive, Chem. Eng. Sci. 159 (2017) 131-139.

[18] B. Van Meerbeek, K. Yoshihara, Y. Yoshida, A. Mine, J. De Munck, K.L. Van Landuyt, State of the art of self-etch adhesives, Dent. Mater. 27 (2011) 17-28.

[19] G. Alex, Universal adhesives: the next evolution in adhesive dentistry? Compend. Contin. Educ. Dent. 36 (2015) 15-26.

[20] H. Xie, Q. Li, F. Zhang, Y. Lu, F.R. Tay, M. Qian, C. Chen, Comparison of resin bonding improvements to zirconia between one-bottle universal adhesives and tribochemical silica coating which is better? Dent. Mater. 32 (2016) 403-411.

[21] K. Yoshihara, N. Nagaoka, A. Sonoda, Y. Maruo, Y. Makita, T. Okihara, M. Irie, Y. Yoshida, B. Van Meerbeek, Effectiveness and stability of silane coupling agent incorporated in 'universal' adhesives, Dent. Mater. 32 (2016) 1218-1225.

[22] C. Chen, L.N. Niu, H. Xie, Z.Y. Zhang, L.Q. Zhou, K. Jiao, J.H. Chen, D.H. Pashley, F.R. Tay, Bonding of universal adhesives to dentine-old wine in new bottles? J. Dent. 43 (2015) 525-536.

[23] Z.Y. Zhang, F.C. Tian, L.N. Niu, K. Ochala, C. Chen, B.P. Fu, X.Y. Wang, D.H. Pashley, F.R. Tay, Defying ageing: an expectation for dentine bonding with universal adhesives? J. Dent. 45 (2016) 43-52.

[24] P. Saikaew, A.F. Chowdhury, M. Fukuyama, S. Kakuda, R.M. Carvalho, H. Sano, The effect of dentine surface preparation and reduced application time of adhesive on bonding strength, J. Dent. 47 (2016) 63-70.

[25] F. Lutz Krejci, In-vitro test results of the evaluation of dental restoration systems Correlation with in-vivo results, Schweiz. Monatsschr. Zahnmed. 100 (1990) 1445-1449.

[26] G.C. Martins, A. Sánchez-Ayala, P.H. D'Alpino, A.L. Calixto, J.C. Gomes, O.M. Gomes, Interfacial integrity of bonded restorations with self-etching adhesives: water storage and thermo-mechanical cycling, Eur. J. Dent. 6 (2012) 169-177.

[27] D.H. Pashley, R.M. Carvalho, H. Sano, M. Nakajima, M. Yoshiyama, Y. Shono, C.A. Fernandes, F.R. Tay, The microtensile bond test: a review, J. Adhes. Dent. 1 (1999) 299-309.

[28] S. Lydersen, V. Pradhan, P. Senchaudhuri, P. Laake, Choice of test for association in small sample unordered r x c tables, Stat. Med. 26 (2007) 4328-4343.

[29] F.R. Tay, D.H. Pashley, M. Yoshiyama, Two modes of nanoleakage expression in single-step adhesives, J. Dent. Res. 81 (2002) 472-476.

[30] A. Destainville, E. Champion, D. Bernache-Assollant, E. Laborde, Synthesis, characterization and thermal behaviour of apatitic tricalcium phosphate, Mater. Chem. Phys. 80 (2003) 269-277.

[31] M. Markovic, B.O. Fowler, M.S. Tung, Preparation and comprehensive characterization of a calcium hydroxyapatite reference material, J. Res. Natl. Inst. Stand. Technol. 109 (2004) 553-568.

[32] F.R. Tay, D.H. Pashley, Aggressiveness of contemporary self-etching systems. I: depth of penetration beyond dentin smear layers, Dent. Mater. 17 (2001) 296-308.

[33] S.S. Oliveira, M.K. Pugach, J.F. Hilton, L.G. Watanabe, S.J. Marshall, G.W. Marshall Jr., The influence of the dentin smear layer on adhesion: a self-etching primer vs. a total-etch system, Dent. Mater. 19 (2003) 758-767.

[34] J. De Munck, K. Van Landuyt, M. Peumans, A. Poitevin, P. Lambrechts, M. Braem, B. Van Meerbeek, A critical review of the durability of adhesion to tooth tissue: methods and results, J. Dent. Res. 84 (2005) 118-132.

[35] M. Toledano, M. Yamauti, E. Osorio, F. Monticelli, R. Osorio, Characterization of micro-and nanophase separation of dentin bonding agents by stereoscopy and atomic force microscopy, Microsc. Microanal. 18 (2012) 279-288.

[36] K.L. Van Landuyt, J. De Munck, J. Snauwaert, E. Coutinho, A. Poitevin, Y. Yoshida, S. Inoue, M. Peumans, K. Suzuki, P. Lambrechts, B. Van Meerbeek, Monomersolvent phase separation in one-step self-etch adhesives, J. Dent. Res. 84 (2005) 183-188.

[37] S. Sauro, F. Mannocci, M. Toledano, R. Osorio, I. Thompson, T.F. Watson, Influence of the hydrostatic pulpal pressure on droplets formation in current etch-and-rinse and self-etch adhesives: a video rate/TSM microscopy and fluid filtration study, Dent. Mater. 25 (2009) 1392-1402.

[38] K.L. Van Landuyt, J. Snauwaert, J. De Munck, E. Coutinho, A. Poitevin, Y. Yoshida, K. Suzuki, P. Lambrechts, B. Van Meerbeek, Origin of interfacial droplets with onestep adhesives, J. Dent. Res. 86 (2007) 739-744.

[39] Q. Ye, Y. Wang, P. Spencer, Nanophase separation of polymers exposed to simulated bonding conditions, J. Biomed. Mater. Res. B Appl. Biomater. 88 (2009) 339-348.

[40] F. Abedin, Q. Ye, R. Parthasarathy, A. Misra, P. Spencer, Polymerization behavior of hydrophilic-rich phase of dentin adhesive, J. Dent. Res. 94 (2015) 500-507.

[41] S. Ören, T. Caykara, Ö. Kantoglu, O. Güven, Effect of pH, ionic strength, and temperature on uranyl ion adsorption by poly (N-vinyl 2-pyrrolidone-g-tartaric acid) hydrogels, J. Appl. Polym. Sci. 78 (2000) 2219-2226.

[42] G.S. Chauhan, A. Kumar, A study in the uranyl ions uptake on acrylic acid and acrylamide copolymeric hydrogels, J. Appl. Polym. Sci. 110 (2008) 3795-3803.

[43] K.L. Van Landuyt, J. Snauwaert, M. Peumans, J. De Munck, P. Lambrechts, B. Van Meerbeek, The role of HEMA in one-step self-etch adhesives, Dent. Mater. 24 (2008) 1412-1419.

[44] A.F. Reis, M.T. Oliveira, M. Giannini, M.F. De Goes, F.A. Rueggeberg, The effect of organic solvents on one-bottle adhesives' bond strength to enamel and dentin, Oper. Dent. 28 (2003) 700-706.

[45] F.R. Tay, D.H. Pashley, Water treeing-a potential mechanism for degradation of dentin adhesives, Am. J. Dent. 16 (2003) 6-12.

[46] F.R. Tay, D.H. Pashley, B.I. Su, N. Hiraishi, C.K.Y. Yiu, Water treeing in simplified dentin adhesives-deja vu? Oper. Dent. 30 (2005) 561-579.

[47] M. Hashimoto, S. Ito, F.R. Tay, N.R. Svizero, H. Sano, M. Kaga, D.H. Pashley, Fluid movement across resin-dentin interface during and after bonding, J. Dent. Res. 83 (2004) 843-848.

[48] F.R. Tay, D.H. Pashley, M.C. Peters, Adhesive permeability affects composite coupling to dentin treated with a self-etch adhesive, Oper. Dent. 28 (2003) 610-621.

[49] M.H. Mahdan, M. Nakajima, R.M. Foxton, J. Tagami, Combined effect of smear layer characteristics and hydrostatic pulpal pressure on dentine bond strength of HEMA-free and HEMA-containing adhesives, J. Dent. 41 (2013) 861-871.

[50] L. Mair, P. Padipatvuthikul, Variables related to materials and preparing for bond strength testing irrespective of the test protocol, Dent. Mater. 26 (2010) e17-23.

[51] H.M. Rêgo, T.S. Alves, E. Bresciani, L.N. Niu, F.R. Tay, C.R. Pucci, Can long-term dentine bonding created in real life be forecasted by parameters established in the laboratory? Sci. Rep. 6 (2016) 37799.

[52] P.M. Scaffa, L. Breschi, A. Mazzoni, C.M. Vidal, R. Curci, F. Apolonio, P. Gobbi, D.H. Pashley, L. Tjäderhane, I.L. Tersariol, F.D. Nascimento, M.R. Carrilho, Codistribution of cysteine cathepsins and matrix metalloproteases in human dentin, Arch. Oral Biol. 74 (2017) 101-107.

[53] R. Seseogullari-Dirihan, F. Apollonio, A. Mazzoni, L. Tjaderhane, D.H. Pashley, L. Breschi, A. Tezvergil-Mutluay, Use of crosslinkers to inactivate dentin MMPs, Dent. Mater. 32 (2016) 423-432.

[54] Encyclopedia. Definition Of: Amara's Law, PC Magazine, 2017 (Accessed 04 November 2017). 